\title{
Brown dwarf formation by binary disruption
}

\author{
S. P. Goodwin ${ }^{1}$ and A. Whitworth ${ }^{2}$ \\ 1 Department of Physics \& Astronomy, University of Sheffield, Hicks Building, Hounsfield Road, Sheffield, S3 7RH, UK \\ e-mail: S.Goodwin@sheffield.ac.uk \\ 2 School of Physics \& Astronomy, Cardiff University, The Queens Buildings, 5 The Parade, Cardiff, CF24 3AA, Wales, UK \\ e-mail: A.Whitworth@astro.cf.ac.uk
}

Received 14 November 2006 / Accepted 27 February 2007

\begin{abstract}
Context. The principal mechanism by which brown dwarfs form, and its relation to the formation of higher-mass (i.e. hydrogenburning) stars, is poorly understood.

Aims. We advocate a new model for the formation of brown dwarfs.

Methods. In this model, brown dwarfs are initially binary companions, formed by gravitational fragmentation of the outer parts $(R \gtrsim 100 \mathrm{AU})$ of protostellar discs around low-mass hydrogen-burning stars. Most of these binaries are then gently disrupted by passing stars to create a largely single population of brown dwarfs and low-mass hydrogen-burning stars.

Results. This idea is consistent with the excess of binaries found in low-density pre-main sequence populations, like that in Taurus, where they should survive longer than in denser clusters.

Conclusions. If brown dwarfs form in this way, as companions to more massive stars, the difficulty of forming very low-mass prestellar cores is avoided. Since the disrupted binaries will tend to be those involving low-mass components and wide orbits, and since disruption will be due to the gentle tides of passing stars (rather than violent $N$-body interactions in small- $N$ sub-clusters), the liberated brown dwarfs will have velocity dispersions and spatial distributions very similar to higher-mass stars, and they will be able to retain discs, and thereby to sustain accretion and outflows. Thus the problems associated with the ejection and turbulence mechanisms can be avoided. This model implies that most, possibly all, stars and brown dwarfs form in binary or multiple systems.
\end{abstract}

Key words. stars: formation - stars: binaries: general - stars: low-mass, brown dwarfs

\section{Introduction}

Brown dwarfs were first observed unambiguously over ten years ago (Rebolo et al. 1995; Nakajima et al. 1995; Oppenheimer et al. 1995). In the subsequent decade it has become clear that brown dwarfs are very numerous, possibly comparable in number to hydrogen-burning stars (Luhman et al. 2007). However, it is still not clear how brown dwarfs form, nor how brown dwarfs relate to planets and hydrogen-burning stars.

The two most frequently invoked theories for brown dwarf formation are "ejection", where a stellar embryo is ejected from a small- $N$ cluster, before it can grow above $0.075 M_{\odot}$ by accretion from its natal core (e.g. Reipurth \& Clarke 2001; Bate et al. 2002; Goodwin et al. 2004a,b; Delgado Donate et al. 2004) and "turbulence", where very low-mass prestellar cores are formed by convergent flows and collapse to form brown dwarfs in isolation (e.g. Padoan \& Nordlund 2002, 2004). A number of other brown dwarf formation mechanisms have been proposed, for example disc fragmentation (Boffin et al. 1998; Watkins et al. 1998a,b; Whitworth \& Stamatellos 2006), and photoerosion of high-mass cores overrun by HII regions (Whitworth \& Zinnecker 2004). However, "ejection" and "turbulence" are the two most popular theories (see Whitworth \& Goodwin 2005, and Whitworth et al. 2007, for reviews of brown dwarf formation theory).

In this paper we review the observational properties of brown dwarfs and low-mass hydrogen-burning stars, and rehearse some of the problems associated with the two main formation theories.
We then present a new theory of brown dwarf formation which avoids these problems.

\section{The properties of brown dwarfs and low-mass hydrogen-burning stars}

There appears to be no good reason why brown dwarfs should form in a different way from low-mass hydrogen-burning stars, and this contention is supported by the observation that the statistical properties of brown dwarfs form a continuum with those of low-mass hydrogen-burning stars, across the divide at $0.075 M_{\odot}$. For example, the stellar Initial Mass Function is continuous across the divide. It peaks at $M_{\star} \sim 0.3 M_{\odot}$ (Kroupa 2002; Chabrier 2003), and at least $20 \%$ of greater than planetary mass objects are brown dwarfs (Luhman et al. 2007). Similarly, the binary statistics of brown dwarfs form a continuum with those of low-mass hydrogen-burning stars: as primary mass is decreased across the divide, the binary fraction decreases, the mean semi-major axis and the logarithmic range of semi-major axes decrease, and the mean mass ratio increases towards unity (Burgasser et al. 2006). In clusters, the radial velocity dispersions and spatial distributions of brown dwarfs and low-mass hydrogen-burning stars are indistinguishable (Briceño et al. 2002). Finally, brown dwarfs are observed to have infrared excesses, indicative of discs (Muench et al. 2001; Natta \& Testi 2001; Jayawardhana et al. 2003; Mohanty et al. 2004; Scholz et al. 2006) and emission lines indicative of both on-going 
accretion (e.g. Scholz \& Eislöffel 2004) and outflows (e.g. Fernández \& Comerón 2001; Natta et al. 2004; Whelan et al. 2005), just like hydrogen-burning stars; the ratio of disc mass to stellar mass appears to be approximately constant, and the accretion rate appears to scale with stellar mass squared across the divide (Muzerolle et al. 2003, 2005).

\section{3. "Ejection" versus "turbulence"}

Whitworth et al. (2007) have stressed that all proposed brown dwarf formation mechanisms probably produce at least some brown dwarfs. They emphasise that it is important to examine what fraction of brown dwarfs are formed by each mechanism. In this section we argue that the two most popular mechanisms "ejection" and "turbulence" - both face significant problems as the dominant mode of brown dwarf formation. These problems then lead us in the next section to propose a somewhat different mechanism which we believe may make a significant contribution to brown dwarf formation.

\subsection{Ejection}

Reipurth \& Clarke (2001) have proposed that brown dwarfs are formed when a dense core collapses and fragments to form a small- $N$ sub-cluster, and then one (or more) of the resulting protostellar embryos is ejected from the natal core, before it can accrete enough mass to reach the hydrogen-burning limit. Once ejected, an embryo is detached from the reservoir of material it might otherwise have accreted, and its mass is essentially constant. In this mechanism, the cores which spawn brown dwarfs must have sufficient mass to also spawn two or three other stars. Therefore it is not necessary to invoke the formation of very lowmass prestellar cores. Low-mass hydrogen burning stars will be ejected from cores in the same way, but since the probability of ejection decreases with increasing mass, brown dwarfs will be ejected from cores preferentially and/or sooner. It seems inescapable that this mechanism operates in nature. However, there are a number of observations which suggest that it may not be the dominant formation mechanism for brown dwarfs.

First, if brown dwarfs and low-mass hydrogen-burning stars are formed by the ejection mechanism, they should have a larger velocity dispersion - and hence also in time a wider spatial extent - than more massive stars born in the same cluster. The effect is not necessarily as dramatic as originally predicted by Reipurth \& Clarke (2001), because typical ejection velocities are at most a few $\mathrm{km} \mathrm{s}^{-1}$, and therefore comparable with the velocity dispersion between neighbouring cores and the velocity dispersion for more massive stars (Bate et al. 2003; Goodwin et al. 2004a,b). However, there should be a high-velocity tail to the distribution of ejection velocities, yielding an extended halo of brown dwarfs, and this is not seen in Taurus (Briceño et al. 2002; Luhman 2006) or Chamaeleon (Joergens 2006).

Second, it appears that a significant fraction of brown dwarfs are attended by massive, extended discs, which sustain long-lived accretion and outflows, just like more massive stars (Muench et al. 2001; Natta \& Testi 2001; Muzerolle et al. 2003; Scholz \& Eisloeffel 2004; Natta et al. 2004; Scholz et al. 2006). This is hard to reconcile with the ejection mechanism. In simulations of core collapse and fragmentation, ejected brown dwarfs do occasionally retain significant discs (see Whitworth et al. 2007), but too infrequently to explain the observations.

Third, the ejection of brown dwarfs and low-mass hydrogenburning stars quickly hardens the system of stars remaining near the centre of the natal core. Simulations suggest that the collapse and fragmentation of a core usually leads to the formation of a small- $N$ sub-cluster; and then ejection of the lower-mass members of the sub-cluster typically reduces it rather quickly to a binary system, often with quite a small separation, $\$ 10 \mathrm{AU}$ (e.g. Goodwin et al. 2004a,b; Goodwin \& Kroupa 2005; Umbreit et al. 2005). As a consequence of this early hardening, the binary system then normally evolves towards equal-mass components. This is because the material accreting later onto the binary system tends to have increasing angular momentum, and therefore it can be accommodated more easily by a low-mass secondary, which pushes the mass ratio, $q$, up towards unity (Whitworth et al. 1995). Whilst close companions are more likely to be of more equal mass (e.g. Duquennoy \& Mayor 1991; Mazeh et al. 1992), the observed trend is not as extreme as predicted by the standard ejection scenario (see Goodwin et al. 2004b; Goodwin et al. 2007).

\subsection{Turbulence}

Padoan \& Nordlund $(2002,2004)$ have proposed that brown dwarfs are formed from the collapse of very low-mass prestellar cores, i.e. a scaled-down version of the formation of more massive hydrogen-burning stars (André et al. 2000; Ward-Thompson et al. 2007). This proposal is supported by the fact that the mass function for prestellar cores appears to echo the shape of the stellar initial mass function, at least at high and intermediate masses (Motte et al. 1998, 2001; Testi \& Sargent 1998; Johnstone 2000; Nutter \& Ward-Thompson 2007). From this it is presumed that the collapse and fragmentation of a prestellar core having mass $M_{\text {CORE }}$ proceeds in a statistically self-similar manner, spawning the same mix of scaled stellar masses $\left(M_{\star} / M_{\text {CORE }}\right)$ with the same efficiency $\left(\sum\left\{M_{\star}\right\} / M_{\mathrm{CORE}}\right)$.

If most prestellar cores spawn just one star (e.g. Lada 2006), the shift of the peak - from $1 M_{\odot}$ for the prestellar core mass function (Nutter \& Ward-Thompson 2007) to $0.3 M_{\odot}$ for the stellar Initial Mass Function (Chabrier 2003) - implies a mean efficiency of $\sim 30 \%$ for converting prestellar cores into stars. To form an isolated brown dwarf with mass $M_{\star} \sim 0.03 M_{\odot}$ then requires a prestellar core with mass $M_{\mathrm{CORE}} \sim 0.1 M_{\odot}$, and hence diameter $D_{\mathrm{CORE}} \sim 300 \mathrm{AU}(T / 10 \mathrm{~K})^{-1}$ and column-density $N_{\text {CORE }} \sim 2 \times 10^{24} \mathrm{~cm}^{-2}$. To form such a prestellar core in isolation requires an implausibly well focussed and dense convergent flow, viz. gas with density $n \sim 10^{4} \mathrm{~cm}^{-3}$ flowing inwards (a) inertially at speed $v \sim 1 \mathrm{~km} \mathrm{~s}^{-1}$ and (b) approximately isotropically over a sphere of radius $r \sim 0.01 \mathrm{pc}$, for a time $t \sim 10^{5}$ years (Whitworth et al. 2007). In an interstellar medium where the principal sources of turbulent energy are shear and expansion, it seems very unlikely that these conditions are fulfilled frequently enough to be a major source of brown dwarfs. More fundamentally, the pure turbulence theory predicts explicitly that for every prestellar core with mass $M_{\mathrm{CORE}} \sim 0.1 M_{\odot}$ there are $\sim 20000$ more diffuse, transient - but longer lived - cores of the same mass, which will not collapse to form stars (Nordlund, private communication). Thus although very low-mass prestellar cores might be sufficiently rare and compact to have escaped detection, some of this extensive population of non-prestellar cores should already have been observed. However, to date there are very few candidate brown dwarf-mass cores (Greaves 2005).

If, instead, most prestellar cores collapse and fragment in a self-similar manner to form small- $N$ sub-clusters, the efficiency for converting cores into stars needs to be somewhat higher, $\sim 50 \%$. The observed binary statistics of Sun-like stars then imply that a sub-cluster should only comprise 3 or 4 stars (in order 
to match the overall binary frequency) and should have quite a wide range of masses, $\sigma_{\log _{10}[M]} \sim 0.6$ (in order to reproduce the observed low mass ratios; Goodwin \& Kroupa 2005; Hubber \& Whitworth 2005). However, there is then no explanation for the observed decline in binary frequency with decreasing primary mass, or for any of the other systematic trends with decreasing primary mass (viz. decreasing mean semi-major axis, decreasing logarithmic spread of semi-major axes, increasing mass ratio; e.g. Burgasser et al. 2006).

\section{Binary disruption}

In this section we propose a new mechanism for the formation of brown dwarfs. We hypothesise that brown dwarfs form as distant companions to low-mass stars, in particular M-dwarfs. Such systems are then readily disrupted by the mild perturbations of passing stars at relatively large distances (a few hundred AU or more). The result is a population of single brown dwarfs and low-mass hydrogen-burning stars.

The disruption of these systems will usually be due to a small velocity perturbation induced by a passing star within the overall cluster (rather than the close and violent interactions in a small$N$ system invoked by the standard ejection hypothesis). Also, as the disruption is gentle, both the brown dwarfs and the lowmass hydrogen-burning stars are able to retain their circumstellar discs. The relative velocities of the components after disruption are low, and therefore there is little spatial or kinematical difference between them and the more massive stars in the same cluster.

This picture avoids the problem of having to create many brown dwarf-mass cores, as in the turbulence mechanism. It also avoids the problems of giving some brown dwarfs a noticeably larger velocity dispersion than the other stars in the cluster, stripping circum-brown dwarf discs, and of hardening the surviving binary systems, as in the ejection mechanism.

\subsection{The formation of wide, low-mass binary systems}

This new mechanism for brown dwarf formation requires firstly that brown dwarfs form as distant companions to low-mass hydrogen-burning stars, and secondly that the resulting soft binary systems are gently dissolved by tidal interactions with passing stars.

The simplest way to form such soft binaries is by disc fragmentation. Rafikov (2005), Matzner \& Levin (2005) and Whitworth \& Stamatellos (2006) have shown that it is very difficult for low-mass companions to form by gravitational instability in the inner parts of circumstellar discs. This is because the inner parts of a disc are too warm, and too strongly irradiated by the central star, for proto-fragments to cool radiatively on a dynamical timescale. Thus, even if the disc is Toomre unstable (Toomre 1964), proto-fragments which try to condense out undergo an adiabatic bounce and are then sheared apart (Gammie 2001). However, low-mass companions can condense out at larger radii,

$R \gtrsim 150 \mathrm{AU}\left(\frac{M_{\star}}{M_{\odot}}\right)^{1 / 3}$,

because here optical depths are lower, stellar irradiation is weaker, and proto-fragments are therefore able to cool radiatively fast enough to condense out (Whitworth \& Stamatellos 2006). The minimum mass for condensations in the outer disc is

$M_{\mathrm{MIND}} \sim 0.003 M_{\odot}\left(\frac{M_{\star}}{M_{\odot}}\right)^{-1 / 4}\left(\frac{L_{\star}}{L_{\odot}}\right)^{3 / 8}$,

so such condensations should usually end up with brown dwarf masses $\left(<0.075 M_{\odot}\right)$.

We conclude that soft, low-mass binary systems should form routinely, provided that low-mass stars acquire sufficiently massive and extended circumstellar discs to spawn brown dwarfs in the manner described above (see Whitworth \& Stamatellos (2006) for further details). From a theoretical perspective, this is at least plausible: observational estimates of the mean specific angular momentum, $h$, in a core (Bodenheimer 1995; his Fig. 1) give values $h \gtrsim 10^{21} \mathrm{~cm}^{2} \mathrm{~s}^{-1}$, and, if deposited in orbit around a star with mass $M_{\star} \sim 0.3 M_{\odot}$, this material should end up at radius

$R \sim \frac{h^{2}}{G M_{\star}} \gtrsim 100 \mathrm{AU}$.

The fact that few such discs are observed can be attributed to the likelihood that they fragment on a dynamical timescale. At $R \sim 100 \mathrm{AU}$, this dynamical timescale is $t_{\mathrm{ORBIT}} \sim 2000$ years, and therefore such discs should be very rare. The resulting binary systems are expected to last somewhat longer ( $\sim 5 \mathrm{Myr}$ in a typical cluster; see Sect. 4.2), and indeed there are several known binary systems in which the primary is a K- or M-type star, and the secondary is a brown dwarf (Burgasser et al. 2005).

\subsection{The disruption of wide, low-mass binary systems}

In the mass range $M_{\star}>0.5 M_{\odot}$, not only is the multiplicity significantly higher for pre-main sequence objects in young clusters than for mature stars in the field (see Goodwin \& Kroupa 2005; Goodwin et al. 2007; Duchêne et al. 2007; and references therein), but also most of the excess is at large separations $\gtrsim 100$ AU (compare Mathieu 1994; Patience et al. 2002 and Duchêne et al. 2007 with Duquennoy \& Mayor 1991). As first pointed out by Kroupa (1995a,b), this difference is most easily explained by the dynamical destruction of binaries in their birth clusters. Wide and/or low-mass binaries are especially susceptible to destruction. Hence, starting with an initial binary fraction of unity, Kroupa was able to recover the lower field binary fraction, the separation distribution, and the dependence of these parameters on primary mass, by simulating dynamical interactions in a representative cluster. There would seem to be no other way to reconcile the high binary fraction for pre-main sequence stars, as compared with the field (Goodwin et al. 2007).

For lower masses, $M_{\star}<0.5 M_{\odot}$, the binary fraction in young clusters is poorly constrained; but in the field, the binary fraction for M-dwarfs appears to be significantly lower $(\sim 30 \pm$ $10 \%$; Fischer \& Marcy 1992) than for G-dwarfs ( 50 $\pm 10 \%$; Duquennoy \& Mayor 1991). This has lead Lada (2006) to suggest that, whereas more massive stars usually form in multiples, less massive ones usually form as singles. However, since it is the low-mass systems which are most readily disrupted, and since disruption must already be invoked for higher-mass stars to explain the systematic shift in binary fraction between clusters and the field, it seems much more likely that low-mass stars are also born in multiple systems and are then selectively disrupted by dynamical interactions. 
To estimate the timescale for disruption, in a cluster, consider a binary system with components of mass $M_{1}$ and $M_{2}$, semimajor axis $a$, and hence binding energy $E_{\text {BINDING }}=G M_{1} M_{2} / 2 a$. Using the impulse approximation (Binney \& Tremaine 1987), a star of mass $M_{\star}$ passing at distance $D$ with velocity $v$ delivers a tidal impulse

$\Delta E_{\mathrm{TIDAL}} \sim \frac{4 G^{2} M_{\star}^{3} a}{3 v^{2} D^{3}}$.

The system will be unbound if $\Delta E_{\mathrm{TIDAL}}>E_{\mathrm{BINDING}}$, i.e. if

$D<\left(\frac{G a^{2}}{3 v^{2} M_{1} M_{2}}\right)^{1 / 3} 2 M_{\star}$.

Substituting $a \sim 100 \mathrm{AU}, v \sim 2 \mathrm{~km} \mathrm{~s}^{-1}, M_{1} \sim 0.3 M_{\odot}, M_{2} \sim$ $0.03 M_{\odot}$ and $M_{\star} \sim M_{\odot}$, this gives $D<2000 \mathrm{AU}$. In a cluster with density $n_{\star} \sim 2000 \mathrm{pc}^{-3}$, the rate for such impulsive interactions is $\sim 0.2 \mathrm{Myr}^{-1}$. In other words, a binary with $M_{1} \sim 0.3 M_{\odot}$, $M_{2} \sim 0.03 M_{\odot}$ and $a \sim 100 \mathrm{AU}$ has a life expectancy of $\sim 5 \mathrm{Myr}$ in such a cluster. (Aguilar \& White (1985) have shown that, even in situations like this, where the orbital velocities of the binary components are comparable with the velocity dispersion of the stars in the cluster, the impulse approximation gives reliable results.)

We conclude that tidal disruption of wide low-mass binaries will result in a growing population of single brown dwarfs and low-mass hydrogen-burning stars. The gentle nature of the disruption will allow the separated binary components to retain circumstellar discs, and hence to sustain accretion and outflows. The disruption velocities will be comparable with the orbital velocities $\left(\lesssim 1 \mathrm{~km} \mathrm{~s}^{-1}\right)$, and hence less than - or on the order of - the velocity dispersion in the cluster. Consequently, brown dwarfs will have essentially the same kinematics and the same spatial distribution as more massive stars.

Bouy et al. (2006) have tentatively identified a population of wide, low-mass binaries in the Upper Scorpius OB Association, with separations between $100 \mathrm{AU}$ and $150 \mathrm{AU}$, as required by our model. They note that no such population is observed in the Pleiades, and suggest that binary properties may change with environment. An alternative explanation is that in the Pleiades, with an age $\sim 100 \mathrm{Myr}$, the primordial population of wide, lowmass binaries has all been disrupted; whereas in Upper Sco OB, with an age $\sim 5 \mathrm{Myr}$ and relatively low density, a significant fraction of the primordial population survives.

\subsection{The binary properties of brown dwarfs and very low-mass hydrogen-burning stars}

In the field, the binary frequency is estimated to be $20 \pm 10 \%$ for brown dwarfs ( $\mathrm{L}$ dwarfs), as compared with $30 \pm 10 \%$ for very low-mass hydrogen-burning stars (M dwarfs) and $50 \pm 10 \%$ for Sun-like stars (G dwarfs) (e.g. Burgasser et al. 2006; Basri \& Reiners 2006; Duquennoy \& Mayor 1991; Fischer \& Marcy 1992; Luhman et al. 2007; Reid et al. 2006). There is some evidence to suggest that the binary frequency for brown dwarfs may be significantly higher in clusters, say $40 \pm 10 \%$ (Pinfield et al. 2003; Maxted \& Jeffries 2005; Chappelle et al. 2006; Montagnier et al. 2006), but this claim awaits confirmation. As primary mass increases, the mean binary separation also increases, and the mean mass ratio, $q \equiv M_{2} / M_{1}$, decreases (e.g. Lucas et al. 2005; Kraus et al. 2006).

As an indication of how our model might relate to these statistics, suppose that the primordial binary fraction for
M dwarfs is $100 \%$, so a cluster might start with 80 primordial M-dwarf binaries. We then require that 30 of these primordial binaries survive (predominantly with M-dwarf secondaries), 20 others with M-dwarf secondaries are disrupted, and 30 with brown-dwarfs secondaries are also disrupted. This leaves $30 \mathrm{M}$-dwarf binaries, $70 \mathrm{M}$-dwarf singles, and 30 browndwarf singles - hence an M-dwarf binary fraction of $~ 30 \%$ and a ratio of $10 \mathrm{M}$ dwarfs to every 3 brown dwarfs. In total, 50 primordial binaries (i.e. $\sim 60 \%$ of the original 80 ) have been disrupted, and these will tend to be the wider ones. Whilst the above reckoning is not intended to be definitive, we note that it is consistent with the over-abundance of wide binaries ( $\gtrsim 100 \mathrm{AU})$ observed in young populations, relative to the field (Patience et al. 2002; in particular, their Fig. 4). It is also consistent with the distribution of mass ratios observed in field M-dwarf binaries by Fischer \& Marcy (1992), which is noticeably flatter than for G-dwarfs (provided one excludes very close systems; Mazeh et al. 1992) and therefore favours less extreme mass ratios in the evolved field population.

\subsection{Close brown dwarf-brown dwarf binaries}

There appears to be a significant population of close $(<20 \mathrm{AU})$ brown dwarf-brown dwarf binaries (Basri \& Martín 1999; Close et al. 2003; Pinfield et al. 2003; Maxted \& Jeffries 2005; Burgasser et al. 2006; Luhman et al. 2007). It is unlikely that such binaries are formed by ejection, since brown dwarfs and low-mass hydrogen-burning stars formed by ejection are almost always single. Equally, a brown-dwarf secondary is unlikely to form by fragmentation of a circumstellar disc around a brown-dwarf primary. Firstly, if the disc is sufficiently massive to fragment, then accretion onto the primary is likely to push its mass above the hydrogen-burning limit. Secondly, fragmentation is precluded in the inner parts $(<100 \mathrm{AU})$ of a circumstellar disc, because the gas is too warm and too strongly irradiated by the central star (Whitworth \& Stamatellos 2006), and therefore disc fragmentation cannot produce close binaries. Close brown dwarf-brown dwarf binaries may form by secondary fragmentation, due to the dissociation of molecular hydrogen (see Goodwin et al. 2007; Whitworth et al. 2007). For example, Whitworth \& Stamatellos (2006) suggest that low-mass fragments condensing out in the outer parts ( $\gtrsim 100 \mathrm{AU})$ of a circumstellar disc - as we are suggesting here - may subsequently undergo secondary fragmentation to produce close brown dwarfbrown dwarf binaries. Gentle disruption can then sometimes separate the close brown dwarf-brown dwarf binary from the star at the centre of the disc without destroying the close brown dwarfbrown dwarf binary itself, thereby populating the field with close brown dwarf-brown dwarf binaries. This scenario would explain the observation that brown dwarfs which remain in wide orbits around more massive stars are - modulo small number statistics - much more likely to be in a close binary system with another brown dwarf than are brown dwarfs in the field (Burgasser et al. 2005).

\subsection{Possible observational tests of this model}

This scenario requires that brown dwarfs form in massive, but short-lived discs around low-mass stars. Such a disc is expected to fragment on a dynamical timescale, and so its lifetime is much shorter than that of a class 0 object (see e.g. Ward-Thompson et al. 2007). Observations of such discs will therefore be hard. 
For example, the dynamical timescale for a disc with diameter $\sim 100 \mathrm{AU}$ around a primary with mass $M_{1} \sim 0.3 M_{\odot}$ is $\sim 3000$ years. If we assume that the brown dwarf formation rate is $\sim 0.2$ /year, and that brown dwarf formation is distributed uniformly over the Galactic disc out to $\sim 10 \mathrm{kpc}$, then there is on average only one such disc within $\sim 400 \mathrm{pc}$, and it is also presumably a deeply embedded, low-luminosity source. ALMA should have no difficulty resolving such a source, but finding it and collecting sufficient photons to identify it unambiguously in a crowded and confused region may be more problematic.

The timescale and efficiency of the disruption of wide, lowmass binaries should depend on the density of the parent cluster. Therefore we would expect loose associations to still contain undisrupted systems. Such a population is indeed observed in the upper Sco OB association (Bouy et al. 2006), but not in Taurus (Kraus et al. 2006). However, we note that most star formation in Taurus appears to occur in fairly dense subgroups whose density might be sufficient to disrupt a low-mass, wide binary population. In addition, we note that Taurus appears to be deficient in M-dwarfs and brown dwarfs relative to a "standard" IMF (see Goodwin et al. 2004c and references therein), and this lack of M-dwarfs may explain the lack of brown dwarfs relative to "typical" regions. We plan to conduct a series of $n$-body simulations to test the efficiency of disruption in different environments.

\section{Conclusions}

We propose that there exists a primordial population of wide low-mass binaries, most of which are rapidly but gently disrupted by tidal encounters in clusters, to produce the observed population of single brown dwarfs and low-mass hydrogenburning stars. This primordial population corresponds to the excess of wide binaries found amongst pre-main sequence stars (e.g. Mathieu 1994; Patience 2002). Such wide, low-mass binaries are highly susceptible to disruption by passing stars, and are unlikely to survive long in the dense environment of a young cluster. However, the disruption of such binaries will normally be very gentle, allowing the individual components (brown dwarfs and low-mass hydrogen-burning stars) to retain circumstellar discs, and hence to sustain accretion and outflows. For the same reason, the peculiar velocities of the components after disruption will be low, and therefore they will be hard to distinguish from the more massive stars in the cluster on the basis of their velocity dispersion or spatial distribution.

This model produces a predominantly single population of brown dwarfs and low-mass hydrogen-burning stars, with a continuum of statistical properties, both across the hydrogenburning limit, and towards higher masses. It obviates both the need to produce very low-mass prestellar cores, as in the pure turbulence theory, and the large peculiar velocities generated by the ejection mechanism.

Lada (2006) has suggested, based on the low field binary fraction of M-dwarfs, that most stars (i.e. M-dwarfs) form as single objects. The model we have presented in this paper, however, implies that all stars and brown dwarfs form within binary and multiple systems. In this case, any theory of star formation must produce multiple systems as the norm.

Note added in proof. After acceptance we became aware of the discovery of several very wide M-dwarf-BD binaries in Taurus (Konopacky et al. 2007, [arXiv: astro-ph/0703567] exactly as predicted by this model.
Acknowledgements. The authors would like to thank Adam Kraus for useful discussions. S.P.G. was supported for part of this work by a UKAFF Fellowship. A.P.W.'s work is supported by PPARC (Ref. PPA/G/O/2002/00497).

\section{References}

Aguilar, L. A., \& White, S. D. M. 1985, ApJ, 295, 374

André, P., Ward-Thompson, D., \& Barsony, M. 2000, Protostars and Planets IV, ed. V. Mannings, A. P. Boss, \& S. S. Russell (Tucson: University of Arizona Press), 59

Basri, G., \& Martín, E. L. 1999, AJ, 118, 2460

Basri, G., \& Reiners, A. 2006, AJ, 132, 663

Bate, M. R., Bonnell, I. A., \& Bromm, V. 2002, MNRAS, 332, L65

Bate, M. R., Bonnell, I. A., \& Bromm, V. 2003, MNRAS, 339, 577

Binney, J., \& Tremaine, S. 1987, Galactic Dynamics (Princeton University Press) Bodenheimer, P. 1995, ARA\&A, 33, 199

Boffin, H. M. J., Watkins, S. J., Bhattal, A. S., Francis, N., \& Whitwirth, A. P. 1998, MNRAS, 300, 1189

Bouy, H., Martín, E. L., Brandner, W., et al. 2006, A\&A, 451, 177

Briceño, C., Luhman, K. L., Hartman, L., Stauffer, J. R., \& Kirkpatrick, J. D. 2002, ApJ, 580, 317

Burgasser, A. J., Kirkpatrick, J. D., \& Lowrance, P. J. 2005, AJ, 129, 2849

Burgasser, A. J., Reid, I. N., Siegler, N., et al. 2007, Protostars and Planets V, ed. B. Reipurth, D. Jewitt, \& K. Keil (Tucson: University of Arizona Press), 427

Chabrier, G. 2003, PASP, 115, 763

Chappelle, R. J., Pinfield, D. J., Steele, I. A., Dobbie, P. D., \& Magazzù, A. 2005, MNRAS, 361, 1323

Close, L. M., Siegler, N., Freed, M., \& Biller, B. 2003, ApJ, 587, 407

Delgado-Donate, E. J., Clarke, C. J., \& Bate, M. R. 2004, MNRAS, 347, 759

Duchêne, G., Delgado-Donate, E., Haisch, K. E., Jr., Loinard, L., \& Rodriguez, L. F. 2007, Protostars and Planets V, ed. B. Reipurth, D. Jewitt, \& K. Keil (Tucson: University of Arizona Press), 379

Duquennoy, A., \& Mayor, M. 1991, A\&A, 248, 485

Fernández, M., \& Comerón, F. 2001, A\&A, 380, 264

Fischer, D. A., \& Marcy, G. W. 1992, ApJ, 396, 178

Gammie, C. F. 2001, ApJ, 553, 174

Goodwin, S. P., \& Kroupa, P. 2005, A\&A, 439, 565

Goodwin, S. P., Whitworth, A. P., \& Ward-Thompson, D. 2004a, A\&A, 414, 633

Goodwin, S. P., Whitworth, A. P., \& Ward-Thompson, D., 2004b, A\&A, 423, 169

Goodwin, S. P., Whitworth, A. P., \& Ward-Thompson, D. 2004c, A\&A 419543

Goodwin, S., Kroupa, P., Goodman, A., \& Burkert, A. 2007, Protostars and Planets V, ed. B. Reipurth, D. Jewitt, K. Keil (Tucson: University of Arizona Press), 133

Greaves, J. 2005, AN, 326, 1044

Hubber, D. A., \& Whitworth, A. P. 2005, A\&A, 437, 113

Jayawardhana, R., Ardila, D. R., Stelzer, B., \& Haisch, K. E., Jr. 2003, AJ, 125, 1515

Joergens, V. 2006, A\&A, 448, 655

Johnstone, D., Wilson, C. D., Moriarty-Schieven, G., GiannakopoulouCreighton, J., \& Gregersen, E. 2000, ApJS, 131, 505

Kraus, A. L., White, R. J., \& Hillenbrand, L. A. 2006, ApJ, 649, 306

Kroupa, P. 1995a, MNRAS, 277, 1491

Kroupa, P. 1995b, MNRAS, 277, 1507

Kroupa, P. 2002, Science, 295, 82

Lada, C. J. 2006, ApJ, 640, L63

Lucas, P. W., Roche, P. F., \& Tamura, M. 2005, MNRAS, 361, 211

Luhman, K. L. 2006, ApJ, 645, 676

Luhman, K. L., Joergens, V., Lada, C., et al. 2007, Protostars and Planets V, ed. B. Reipurth, D. Jewitt, K. Keil (Tucson: University of Arizona Press), 443

Mathieu, R. D. 1994, ARA\&A, 32, 465

Matzner, C. D., \& Levin, Y. 2005, ApJ, 628, 817

Maxted, P. F. L., \& Jeffries, R. D. 2005, MNRAS, 362, L45

Mazeh, T., Goldberg, D., Duquennoy, A., \& Mayor, M. 1992, ApJ, 401, 265

Mohanty, S., Jayawardhana, R., Natta, A., et al. 2004, ApJ, 609, L33

Montagnier, G., Segransan, D., Beuzit, J.-L., et al. 2006, A\&A, 460, L19

Motte, F., André, P., \& Neri, R. 1998, A\&A, 336, 150

Motte, F., André, P., Ward-Thompson, D., \& Bontemps, S. 2001, A\&A, 372, L41

Muench, A. A., Alves, J., Lada, C. J., \& Lada, E. A. 2001, ApJ, 558, L51

Muzerolle, J., Hillenbrand, L., Calvet, N., Briceño, C., \& Hartman, L. 2003, ApJ, 592, 266

Muzerolle, J., Luhman, K., Briceño, C., Hartman, L., \& Calvet, N. 2005, ApJ, 625,906

Nakajima, T., Oppenheimer, B. R., Kulkarni, S. R., et al. 1995, Nature, 378, 463 Natta, A., \& Testi, L. 2001, A\&A, 376, L22 
Natta, A., Testi, L., Muzerolle, J., et al. 2004, A\&A, 424, 603

Nutter, D., \& Ward-Thompson, D. 2007, MNRAS, 374, 1413

Oppenheimer, B. R., Kulkarni, S. R., Matthews, K., \& Nakajima, T. 1995, Science, 270, 1478

Padoan, P., \& Nordlund, ̊̊ 2002, ApJ, 576, 870

Padoan, P., \& Nordlund, ̊̊ 2004, ApJ, 617, 559

Patience, J., Ghez, A. M., Reid, I. N., \& Matthews, K., 2002, AJ, 123, 1570

Pinfield, D. J., Dobbie, P. D., Jameson, R. F., et al. 2003, MNRAS, 342, 1241

Rafikov, R. R. 2005, ApJ, 621, L69

Rebolo, R., Zapatero Osorio, M. R., \& Martin, E. L. 1995, Nature, 377, 129

Reid, I. N., Lewitus, E., Allen, P. R., Cruz, K. L., \& Burgasser, A. J. 2006, AJ, 132,891

Reipurth, B., \& Clarke, C. J. 1995, AJ, 122, 432

Scholz, A., \& Eislöffel, J. 2004, A\&A, 419, 249

Scholz, A., Jayawardhana, R., \& Wood, K. 2006, 645, 1498

Testi, L., \& Sargent, A. I. 1998, ApJ, 508, L91

Toomre, A. 1964, ApJ, 139, 1217
Umbreit, S., Burkert, A., Henning, T., Mikkola, S., \& Spurzem, R. 2005, ApJ, 623,940

Ward-Thompson, D., André, P., Crutcher, R., et al. 2007, Protostars and Planets V, ed. B. Reipurth, D. Jewitt, K. Keil (Tucson: University of Arizona Press), 33

Watkins, S. J., Bhattal, A. S., Boffin, H. M. J., Francis, N., \& Whitworth, A. P. 1998a, MNRAS, 300, 1205

Watkins, S. J., Bhattal, A. S., Boffin, H. M. J., Francis, N., \& Whitworth, A. P. 1998b, MNRAS, 300, 1214

Whelan, E. T., Ray, T. P., Bacciotti, F., et al. 2005, Nature, 435, 652

Whitworth, A., Bate, M. R., Nordlund, Å., Reipurth, B., \& Zinnecker, H. 2007, Protostars and Planets V, ed. B. Reipurth, D. Jewitt, \& K. Keil (Tucson: University of Arizona Press), 459

Whitworth, A. P., \& Goodwin, S. P. 2005, AN, 326, 899

Whitworth, A. P., \& Stamatellos, D. 2006, A\&A, 458, 817

Whitworth, A. P., \& Zinnecker, H. 2004, A\&A, 427, 299

Whitworth, A. P., Chapman, S. J., Bhattal, A. S., et al. 1995, MNRAS, 277, 727 